\title{
Optimization Of Combined Economic and Emission Dispatch Problem - A Comparative Study for 30 Bus Systems
}

\author{
R Manoj Kumar.Bavisetti,ME Student ${ }^{1}$, T.Kranthi Kiran,Asst. Professor ${ }^{2}$ \\ ${ }^{1,2}$ (Department of Electrical \&Electronics Engineering, Sir C R R College of Engg, Eluru, India)
}

\begin{abstract}
This paper presents an evolutionary computation (EC) method called Genetic Algorithm (GA) and a metaheuristic algorithm called Ant Colony Search Algorithm (ACSA) to solve the combined Economic and Emission dispatch (EED) problem with transmission losses. Economic Load Dispatch (ELD) and Economic Emission Dispatch (EED) have been applied to obtain optimal fuel cost and optimal emission of generating units, respectively. Combined Economic Emission Dispatch (CEED) problem is obtained by considering both the economy and emission objectives. A real coded GA has been implemented to minimize both the dispatch cost as well as emission while satisfying all the equality and inequality constraints. ACSA is also developed to provide a means of comparison and it is a new cooperative agents approach, which is inspired by the observation of the behaviors of real ant colonies on the topic of ant trail formation and foraging methods. In the ACSA, a set of cooperating agents called "ants" cooperates to find a good solution for economic dispatch problem. The merits of ACSA are parallel search and optimization capabilities. The feasibility of the proposed method is tested on a power system network and the experimental results of both GA and ACSA are compared with the solutions of conventional Lamda iteration method.
\end{abstract}

Keywords : Economic load dispatch, emission reduction, Ant Colony search algorithm, co - operating agents, Genetic algorithm, multi objective optimization.

\section{INTRODUCTION}

The economic load dispatching (ELD) is one of key problems in power system operation and planning. The basic objective of economic load dispatch (ELD) is to schedule the committed generating unit outputs so as to meet the load demand at minimum operating cost, while satisfying all equality and inequality constraints. This makes the ELD problem a large - scale highly constrained non linear optimization problem. The economic emission dispatching (EED) option is an attractive short - term alternative in which the emission in addition to the fuel cost objective is to be minimized.

At present, thermal power plants account for the great majority installations in India. [3] Generally the coal used in thermal plants is of poor quality and high ash. Sulphur dioxide (SO2) and oxides of nitrogen (NOx) are the major emissions from thermal plants due to the combustion of coal which will cause ill effects on human beings as well as on plants and on animals. The Clean Air Act Amendments of 1990 mandates that the electric utility industry reduce its SO2 emission by 10 million tons / year from the 1980 level [4]. The NOx is required to be reduced by 2 million tons/year from the 1980 level. Minimum emission and regulatory agencies of developing countries also in the near future to control and reduce air pollution.

This paper presents GA and ACSA for solving EED problem of an IEEE 30 bus system. The experimental results are compared with the solutions of Lamda iteration method and the computation efficiency is found to be more efficient in ACSA than any other conventional method.

\section{EED PROBLEM ForMUlation}

The objective of Economic Emission Dispatch is to minimize two competing functions, fuel cost and emission, while satisfying several equality and inequality constraints. Therefore it is formulated as follows.

\subsection{Minimization of Fuel Cost}

The generator cost curves are represented by quadratic functions and the total fuel cost $\mathrm{F}(\mathrm{Pg})$ in $(\$ / \mathrm{h})$ can be expressed as

$$
F\left(P_{G}\right)=\sum_{i=1}^{N} a_{i}+b_{i} P_{G_{i}}+c_{i} P_{G_{i}}^{2}
$$

Where $\mathrm{N}$ is the number of generators; $\mathrm{a}_{\mathrm{i}}, \mathrm{b}_{\mathrm{i}}, \mathrm{c}_{\mathrm{i}}$ are the cost coefficients of the $\mathrm{i}_{\text {th }}$ generator and $\mathrm{P}_{\mathrm{G} 1}$ is the real power output of the $\mathrm{I}_{\mathrm{th}}$ generator, $\mathrm{P}_{\mathrm{G}}$ is the vector of real power outputs of generators and defined as

$$
P_{G}=\left[P_{G_{1}}, P_{G_{2}}, \ldots, P_{G_{N}}\right]
$$


2.2 Minimization of Emission

The total emission $\mathrm{E}\left(\mathrm{P}_{\mathrm{G}}\right)$ in $($ ton/h) of atmospheric pollutants such as sulphur oxides $\mathrm{SO} \mathrm{x}$ and nitrogen oxides NO $\mathrm{x}$ caused by the operation of fossil - fueled thermal generation can be expressed as

$$
E\left(P_{G}\right)=\sum_{i=1}^{N} 10^{-2}\left(\alpha_{i}+\beta_{i} P_{G_{i}}+\gamma_{i} P_{G_{i}}^{2}\right)+\xi{ }_{i} \exp \left(\lambda_{i} P_{G_{i}}\right)
$$

Where $\alpha_{\mathrm{i}}, \beta_{\mathrm{i}}, \gamma_{\mathrm{i}}$, and $\lambda_{\mathrm{i}}$ are the coefficients of the $\mathrm{i}_{\mathrm{th}}$ generator emission characteristics.

\subsection{Constraints}

2.3.1 Generation Capacity Constraint:

For stable operation, the real power output of each generator is restricted by lower and upper limits as follows:

$$
P_{G_{i}}^{\min } \leq P_{G_{i}} \leq P_{G_{i}}^{\max }, \quad i=1,2, \ldots, N
$$

\subsubsection{Power Balance Constraints:}

The total electric power generation must cover the total electric power demand $P_{D}$ and the real power loss in transmission lines $\mathrm{P}_{\text {loss }}$, hence

$$
\sum_{i=1}^{N} P_{G_{i}}-P_{D}-P_{\text {loss }}=0
$$

The transmission losses can be evaluated by means B - Matrix method that is taken into account so as to achieve the accurate economic dispatch.

$$
P_{\text {loss }}=\sum_{i=1}^{N} \sum_{j=1}^{N} P_{i} B_{i j} P_{j}+{ }_{i=1}^{N} B_{i 0} P_{i}+B_{00}
$$

Where,

$\mathrm{P}_{\mathrm{j}}$ - The output generation of unit $\mathrm{J}(\mathrm{MW})$

$B_{i j}-$ The $i_{\text {th }}$ element of the loss coefficient square matrix

$\mathrm{B}_{\mathrm{i} 0}-$ The $\mathrm{i}_{\mathrm{th}}$ element of the loss coefficient

$\mathrm{B}_{00}$ - The loss coefficient constraint

2.4 Formulation:

The bi - objective combined economic emission dispatch problem is converted into single optimization problem by introducing price penalty factor $\mathrm{h} 1$ as follows:

$$
\text { Minimize } \Phi=\mathrm{F}+h^{*} \mathrm{E} \$ / \mathrm{hr}
$$

Subjected to the constraints:

$$
\begin{aligned}
& g\left(P_{G}\right)=0 \\
& h\left(P_{G}\right) \leq 0
\end{aligned}
$$

Where $\mathrm{g}$ is the equality constraint representing the power balance, while $\mathrm{h}$ is the inequality constraint representing the generation capacity. The price penalty factor $\mathrm{h}$ blends the emission with fuel cost and is the total operating cost in $\$ / \mathrm{hr}$.

The price penalty factor hi [1] is the ratio between the maximum fuel cost and maximum emission of corresponding generator.

$$
h_{i}=\frac{F\left(P_{G_{i}}^{\max }\right)}{E\left(P_{G_{i}}^{\max }\right)}
$$

$$
\$ / 1 \mathrm{~b} i=1,2, \ldots, N
$$

III. Eed PRoblem Solution BY GA

GA s are inspired by the study of genetics [2]. It is a probabilistic search algorithm that transforms a set of mathematical objects each associated with a fitness function into a new population of offspring objects based on the principle of natural selection, crossover and mutation. In this paper real coded GA (working on floating point or integer) is used to solve this optimization problem. In the GA solution, the output of each free generator is represented by a floating - point number and they are concatenated to form a solution string of $\mathrm{n}$ numbers called chromosome. The flow chart for EED solution using GA is shown in Fig. 1 


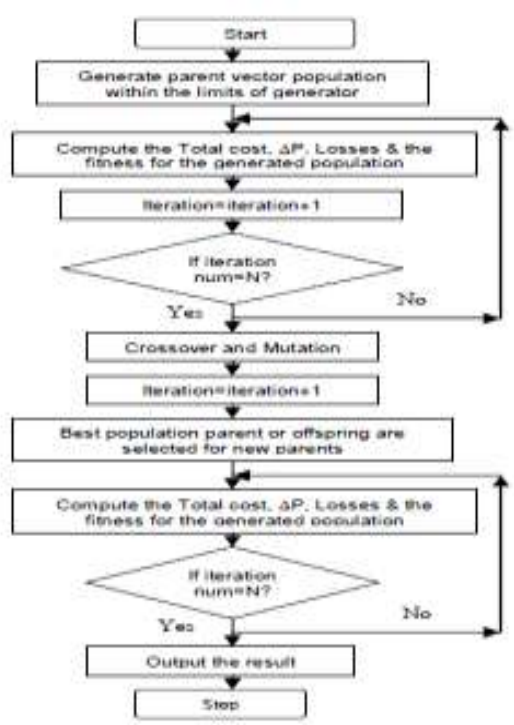

Fig.1.TheFlow chart for EED program using GA

\subsection{Behavior of Real Ants:}

\section{EED PROBLEM SOLUTION BY ACSA}

Ant colony search (ACS) studies are inspired from the behavior of real ant colonies that are used to solve function or combinatorial optimization problems. Ant colony algorithms, to some extent, mimic the behavior of real ants. In fact, real ants are capable of finding the shortest path from food sources to the nest without using visual cues. They are also capable of adapting to changes in the environment. The studies by ethnologists reveal that such capabilities are essentially due to what is called "pheromone tails", which ants use to communicate information among individuals regarding path and to decide where to go [6]. Ants deposit a certain amount of pheromone while walking, and each ant probabilistically prefers to follow a direction rich in pheromone rather than poorer one.

Consider the behavior of ants in finding a new shortest path, once the old one is no longer feasible due to anew obstacle. The process can be clearly illustrated in Fig. 2 (a) where, ants are moving on a straight line that connects a food source to their nest. Ants deposit pheromone while walking and probabilistically prefer to follow a direction rich in pheromone.

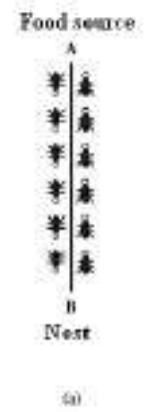

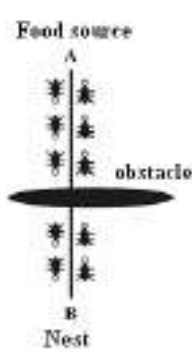

in

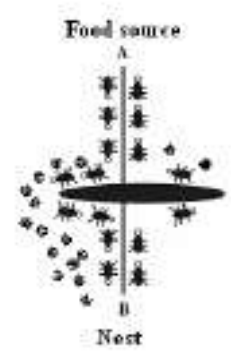

()

Fig. 2 an example of the real ants behavior.

Fig. 2. (a) Real ants follow a path between nest and food source.

(b) An obstacle appears on the path: ants choose whether to turn left or right with equal probability.

(c) Pheromone is deposited more quickly on the shorter path. All ants have chosen the shorter path.

This behavior can be explained how ants can find the shortest path that reconnects a line that is broken by an obstacle in Fig. 2 (b). On introducing, those ants are just in front of the obstacle and they have to choose between turning right or left. Half the ants decide to turn right and the other half decide to turn left. A similar situation arises on the other side of the obstacle. Ants choosing the shorter path will more rapidly reconstitute the interrupted pheromone trail compared with those choosing the longer path. Thus, the compared with those choosing the longer path. Thus, the shorter path will receive a greater amount of pheromone per time unit and, in turn, a larger number of ants will choose the shorter path. Due to this positive feed back, all the ants will rapidly choose the shorter path. Fig.2(C). All ants move at approximately the same speed and deposit a pheromone trail at approximately the same rate. The time to go round the longer side of an obstacle is greater 
than the shorter. This makes the pheromone trail accumulate more quickly on the shorter side. Ants prefer higher pheromone trail levels causing this accumulation to build up still faster on the shorter path.

\subsection{Ant Colony Search Algorithm:}

The Algorithm is described as follows [6]

4.2.1 Initialize the ACS - based optimization problem. Construct searching space including the states and stages of the optimization problem and set the ant number and the parameters of the ACO algorithm.

4.2.2 Find the paths for the ant dispatch. Each ant chooses the states to complete a tour according to a probabilistic state transition rule. Ants prefer to move to states, which are connected by shorted edges with high amount of pheromone. Once all ants have finished their tour, some fitness functions of the optimization problem can be used to evaluate the performance of the ants.

4.2.3 Update the pheromone of edges between each stage. The pheromone trail of each edge will evaporate over time, i.e., it looses intensity if no more pheromone is laid down by other ants. For those edges that ants traveled in this iteration, their pheromone intensity can be updated by the pheromone - updating rule. Global and local pheromone updating rules are generally used to update the pheromone trail.

\subsubsection{Local Updating Rule:}

While constructing its tour, each ant modifies the pheromone by the local updating rule. This can be written below:

$$
\tau(i, j)=(1-\rho) \tau(i, j)+\rho \tau_{0}
$$

$\tau_{\mathrm{o}}$ - the initial pheromone value.

$\rho$ - Is a heuristically defined parameter.

The local updating rule is intended to shuffle the search process. Hence, the desirability of paths can be also explored later by other ants. The search space can be therefore extended.

4.2.3.2 Global Updating Rule:

When tours are completed, the global updating rule is applied to edges belonging to the best and tour this rule is intended to provide a greater amount of pheromone to shorter tours, which can be expressed below

$$
\tau(\mathrm{i}, \mathrm{j})=(1-\sigma) \tau(\mathrm{i}, \mathrm{j})+\sigma \delta-1
$$

$\delta$ - Is the distance of the globally best tour from the beginning of the trial.

$\sigma$ - Is the pheromone decay parameter.

This rule is intended to make the search more directed. Therefore, the capability of finding the optimal solution can be enhanced through this rule in the problem solving process.

4.2.4 Define the Convergence criteria of the problem. This process is iterated until the tour counter reaches the maximum pre - defined number of iterations or all ants make the same tours.

4.3 ACS State Transition Rule:

$$
\left.\eta{ }_{[i}\right]=\frac{S U[i]}{\sum_{m} S U[m]}
$$

The state transition rule used by ant system is called a random - proportional rule and is given in Equation. (8). It gives the probability with which ant $\mathrm{k}$ in city "p" chooses to move to the city "k".

$$
P_{p k}=\frac{\left(\tau_{p k}\right)^{\alpha}\left(\eta_{k}\right)^{\beta}}{\sum_{i}\left(\tau_{\mu k}\right)^{\alpha}(\eta)^{\beta}}
$$

The quantities and are parameters of the algorithm. The quantity, the pheromone concentration. The pheromone level of each pair of sections is updated at the end of each trial when the ant has generated a valid dispatch of generated powers. During the course of defining a solution, each ant maintains a data structure, called the tabu list. This list is merely the set of all paths that have already been traversed by it. The tabu list is necessary to prevent the ant from including the same path again, producing redundancies, and often, invalid solutions. In most implementations, the solution produced in each iteration can be extracted directly from this set.

Pheromone evaporation plays an inverse role to pheromone deposition. Pheromones are subject to dissipation even in real ants. In ACO, pheromones are evaporated from the ant trails to prevent them from increasing monotonically to undesirable levels. Evaporation is controlled by a parameter, the evaporation rate that controls how quickly trails are updated. A very slow evaporation rate would make the algorithm converge towards good neighborhoods in the solution space more slowly. But on the other hand, a very high rate of evaporation would result in an undue amount of trail being deposited along the path of any ant, thereby causing subsequent ants to follow closely, if not exactly, the same path. 


\section{TEST CASE}

The details of the IEEE 30 bus test case system is shown in fig. 4. The values of the fuel and emission co - efficient of the generators are given in Table I and II respectively.

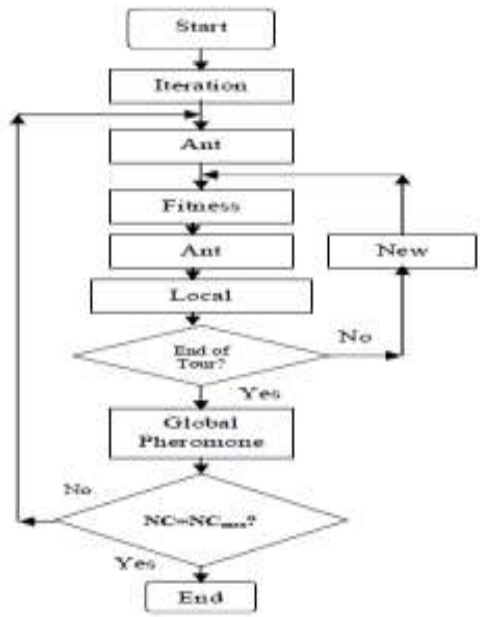

Fig. 3 The Flow Chart of ACSA for EED program

Table I

GENERATOR COST FUNCTIONS OF IEEE 30 BUS SYSTEMS

\begin{tabular}{|c|c|c|c|c|c|}
\hline \multirow{2}{*}{$\begin{array}{l}\text { UNIT } \\
\text { NO. }\end{array}$} & \multirow{2}{*}{$\frac{P_{\min }}{(\mathrm{MW})}$} & \multirow{2}{*}{$\begin{array}{c}\mathrm{P}_{\max } \\
(\mathrm{MW})\end{array}$} & \multicolumn{3}{|c|}{ COST COEFFICIENT } \\
\hline & & & $\begin{array}{c}\mathrm{a}_{\mathrm{i}} \\
(\$ / \mathrm{hr})\end{array}$ & $\begin{array}{c}\mathrm{b}_{\mathrm{i}} \\
(\$ / \mathrm{MW} / \mathrm{hr})\end{array}$ & $\begin{array}{c}\mathrm{C}_{\mathrm{i}} \\
\left(\mathrm{S} / \mathrm{MW}^{2} / \mathrm{hr}\right)\end{array}$ \\
\hline 1 & 50 & 200 & 0 & 2.00 & 0.00375 \\
\hline 2 & 20 & 80 & 0 & 1.75 & 0.01750 \\
\hline 5 & 15 & 50 & 0 & 1.00 & 0.06250 \\
\hline 8 & 10 & 35 & 0 & 3.25 & 0.00834 \\
\hline 11 & 10 & 30 & 0 & 3.00 & 0.00250 \\
\hline 13 & 12 & 40 & 0 & 3.00 & 0.00250 \\
\hline
\end{tabular}

Table II

EMISSION CO EFFICIENTS OF IEEE 30 BUS SYSTEMS

\begin{tabular}{|c|c|c|c|c|c|}
\hline \multirow{2}{*}{$\begin{array}{l}\text { UNIT } \\
\text { NO. }\end{array}$} & $\begin{array}{c}\alpha_{\mathrm{i}} \\
\text { (tons/h } \\
\mathbf{r}\end{array}$ & $\begin{array}{c}\boldsymbol{\beta}_{\mathrm{i}} \\
\text { (tons/ } \\
\mathrm{MWhr}\end{array}$ & $\begin{array}{c}\gamma_{\mathrm{i}} \\
\text { (tons/ } \\
\mathrm{MW}^{2} \\
\mathrm{~h} \text { ) }\end{array}$ & $\begin{array}{c}\boldsymbol{\xi}_{\mathrm{i}} \\
\text { (tons/ } \\
\mathrm{hr} \text { ) }\end{array}$ & $\begin{array}{c}\lambda_{\mathrm{i}} \\
\text { (tons/ } \\
\text { MWhr) }\end{array}$ \\
\hline G1 & 4.091 & -5.094 & 6.490 & $2.0 \mathrm{E}^{-4}$ & 2.857 \\
\hline G2 & 2.543 & -6.047 & 5.638 & $5.0 \mathrm{E}^{-4}$ & 3.333 \\
\hline G3 & 4.258 & -5.094 & 4.586 & $1.0 \mathrm{E}^{-6}$ & 8.000 \\
\hline G4 & 5.326 & -3.550 & 3.380 & $2.0 \mathrm{E}^{-3}$ & 2.000 \\
\hline G5 & 4.258 & -5.094 & 4.586 & $1.0 \mathrm{E}^{-6}$ & 8.000 \\
\hline G6 & 6.131 & -5.555 & 5.151 & $1.0 \mathrm{E}^{-5}$ & 6.667 \\
\hline
\end{tabular}

VI. Numerical Results

The feasibility of the proposed method is tested on IEEE 30 bus system shown in fig. 4. The total generation cost and emission of the 30 bus system is shown in Table $\mathrm{V}$.

The details of the IEEE 30 bus test case system are explained as follows:

Case 1: The ED by Lambda iteration method

The total generation production costs and emission of case 1 are shown in Table V. The results show that the Lambda iteration method consumes the least generation cost. The total operating cost calculated by Lambda iteration method for a demand of $2.834 \mathrm{MW}$ is $644.25 \$ / \mathrm{hr}$. 


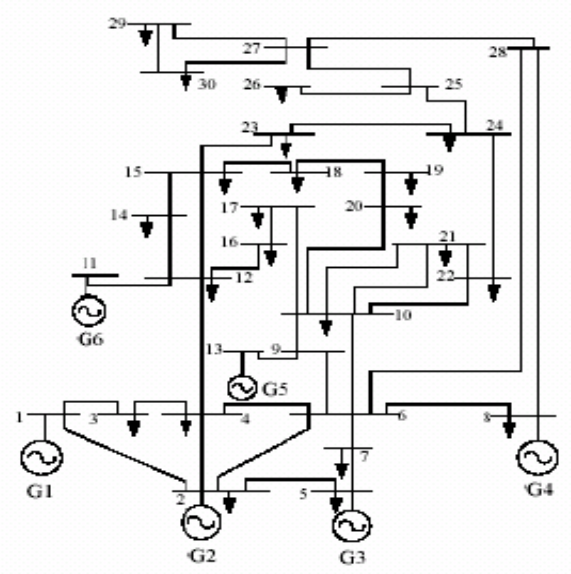

Fig.4 Single line diagram of IEEE 30 bus test system

Table V.MINIMUM SOLUTION OF DIFFERENTS METHODS- IEEE 30 BUE SYSTEMS

\begin{tabular}{|c|c|c|c|c|c|c|c|c|c|c|c|c|}
\hline \multicolumn{13}{|c|}{ IEEE 30 BUS SYSTEM } \\
\hline \multirow{2}{*}{ Method } & \multirow{2}{*}{$\begin{array}{c}\text { Price } \\
\text { Penalty } \\
\text { Factor } \\
\text { PF } \\
(\$ / b) \\
\end{array}$} & \multicolumn{6}{|c|}{ Generator Powers (MW) } & \multirow{2}{*}{$\begin{array}{c}\text { Total } \\
\text { Loss } \\
\text { PL } \\
\text { (MW) }\end{array}$} & \multirow{2}{*}{$\begin{array}{c}\text { Fuel } \\
\text { Cost FC } \\
\text { (\$/hr) }\end{array}$} & \multirow{2}{*}{$\begin{array}{c}\text { Emission } \\
\text { Output } \\
\text { (llb/hr) }\end{array}$} & \multirow{2}{*}{$\begin{array}{c}\text { Emission } \\
\text { Cost } \\
\mathrm{EC}(\$ / \mathrm{hr})\end{array}$} & \multirow{2}{*}{$\begin{array}{c}\text { Total } \\
\text { Operating } \\
\text { Cost } \\
\text { TC (S/hr) }\end{array}$} \\
\hline & & $P_{1}$ & P2 & $\mathrm{P} 3$ & $\mathrm{P} 4$ & P5 & P6 & & & & & \\
\hline LAMBDA & 48.7954 & 0.2887 & 0.2487 & 0.7618 & 0.5063 & 0.7503 & 0.3011 & 0.2243 & 628.96 & 0.3133 & 15.2902 & 644.25 \\
\hline GA & 48.7954 & 0.3401 & 0.2872 & 0.7892 & 0.6095 & 0.3578 & 0.4502 & 0.1956 & 627.83 & 0.2491 & 12.155 & 639.98 \\
\hline $\mathrm{ACO}$ & 48.7954 & 0.2574 & 0.4326 & 0.3855 & 0.6433 & 0.3243 & 0.4408 & 0.1430 & 625.0218 & 0.1965 & 9.3261 & 634.34 \\
\hline
\end{tabular}

Table VI.BEST SOLUTIONS OBTAINED BY ACSA METHOD- IEEE 30 BUE SYSTEMS

\begin{tabular}{|c|c|c|c|c|c|c|c|c|c|c|c|c|c|}
\hline PF & P1 & P2 & P3 & P4 & P5 & P6 & LOSS & $\mathrm{FC}$ & $\mathrm{E}$ & $\mathrm{EC}$ & $\mathrm{TC}$ & FIT & TIME \\
\hline 48.7954 & 0.4155 & 0.1007 & 0.4068 & 1.0081 & 0.4790 & 0.5761 & 0.1436 & 627.1924 & 0.2140 & 10.8455 & 638.0079 & 0.0016 & 2.0310 \\
\hline 48.7954 & 0.2692 & 0.2686 & 0.9704 & 1.0186 & 0.3776 & 0.0827 & 0.1407 & 624.1170 & 0.2300 & 10.8895 & 635.0065 & 0.0016 & 2.0620 \\
\hline 48.7954 & 0.3463 & 0.5901 & 0.3802 & 1.0159 & 0.2943 & 0.3568 & 0.1430 & 625.8798 & 0.2119 & 10.7731 & 636.6529 & 0.0016 & 2.0620 \\
\hline 48.7954 & 0.4826 & 0.4542 & 0.5218 & 0.8992 & 0.4303 & 0.1884 & 0.1421 & 626.4943 & 0.2052 & 10.8020 & 637.2963 & 0.0016 & 2.2040 \\
\hline 48.7954 & 0.3210 & 0.2114 & 0.4923 & 0.6401 & 0.7297 & 0.5888 & 0.1430 & 627.9248 & 0.1961 & 10.4967 & 638.4215 & 0.0016 & 2.1720 \\
\hline 48.7954 & 0.2686 & 0.4751 & 0.5464 & 0.9494 & 0.1542 & 0.5984 & 0.1431 & 626.7664 & 0.2097 & 9.7822 & 636.5486 & 0.0016 & 2.0780 \\
\hline 48.7954 & 0.2574 & 0.4326 & 0.3855 & 0.6433 & 0.3243 & 0.4408 & 0.1430 & 625.0218 & 0.1965 & 9.3261 & 634.3479 & 0.0016 & 2.0780 \\
\hline
\end{tabular}

Case 2: The ED by real - coding genetic algorithm

The Roulette wheel selection and two points cross over are used. The parameters of GA are set as: Number of bits $=12$, Maximum Number of iterations $=250$, crossover probability $=0.96$ and mutation probability $=0.033$. The results for the best run are presented in Tables $\mathrm{V}$. The results show that GA is capable of finding the near optimal solution but it is not very effective in minimizing the total generation cost. The best total operating cost calculated by Genetic Algorithm Method is 639.98 \$/hr.

Case 3: The ED by the proposed ACSA

ACSA can search the best solution than the Lambda iteration method and the heuristic GA method but, it takes more computing time than the Lambda iteration method. The parameters of ACSA are set as: maximum number of iterations $=100$, number of ants $=6$. The best total operating cost calculated by Ant Colony Search Algorithm method is $636.55 \$ / \mathrm{hr}$ is shown in table VI.

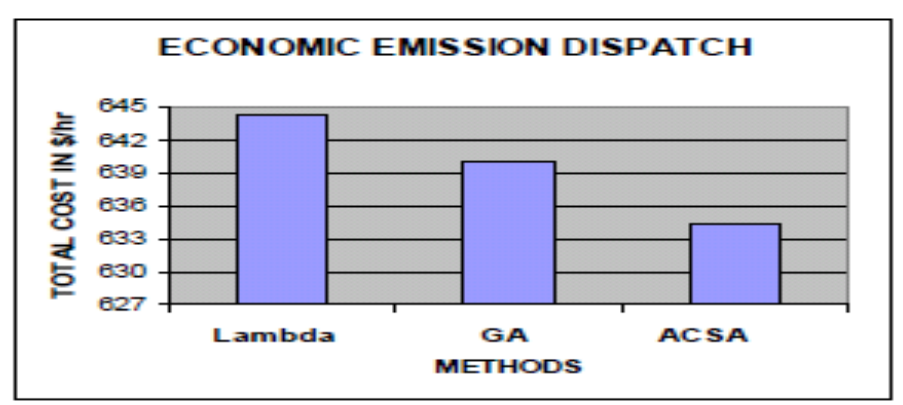

Fig.5Comparision of the results obtained by various methods 
The results obtained by the three methods for a 30 bus system are compared and the comparison is shown in the graphs Fig 5. It shows the merit of ACSA which gives a considerable reduction in the total operating cost and emission calculated in comparison to the cost calculated by Lambda iteration and GA method.

\section{Conclusion}

The results show that GA solution is found to be more optimal than the conventional Lambda iteration method. But GA degrades its performance due to premature convergence and reduced search capability. The solution obtained by ACSA is more accurate compared to the Lambda iteration method and is more optimal compared to Genetic Algorithm method. Thus ACSA is observed to be efficient in terms of minimizing the cost and thermal emission solution but it takes more computing time.

\section{REFERENCES}

[1] Hiroyuki Mori Takuya Horiguchi (1993), ‘A Genetic Algorithm Based Approach to Economic Load Dispatching'. Proceedings of IEEE, Vol 01, No. 1, pp $145-150$.

[2] Bakirtzis.A, Petridis.V and Kazarlis.s (1994), 'Genetic Algorithm solution to the economic dispatch problem'. Proceedings of IEEE, Vol 141, No. , pp.377 - 382 .

[3] A.A.El - Keib, H.Ma and J.L. Hart(1994),'Economic Dispatch in view of the clean air act of 1990', IEEE Transactions on power systems, Vol.9, No. 2, pp.972-978.

[4] Maheswarapu sydulu(1999), 'A Very fast and effective non iterative - logic based algorithm for economic dispatch of thermal units', proceedings of EEE tencon.

[5] Venkatesh.P, Gnanadass.R, and Narayana Prasad Padhy (2003), 'Comparison and Application of Evolutionary Programming Techniques to Combined Economic Emission Dispatch with Line Flow Constraints', IEEE Transactions on power systems, Vol.18, No. 2. pp $688-697$.

[6] Thanathip Sum - im (2004), 'Economic Dispatch by Ant Colony Search Algorithm', Proceedings of the IEEE Conference on Cybernetics and intelligent Systems, Vol.04, No. 4, pp.416- 421. 